\title{
Establishment of a Clinical Gestational Carrier Program: Medical, Ethical, Legal and Policy Issues
}

\author{
Ginny Ryan, M.D. ${ }^{1}$ \\ Key Words: Gestational Carrier Program, IVF, lowa, ethics, surrogacy
}

\begin{abstract}
Pressures to expand, advancing technologies, and increased public exposure of in vitro fertilization (IVF) compels ongoing evaluation of the medical, ethical, and legal proprietary of this high tech infertility treatment, especially when used to build nontraditional families. Recently, the University of lowa's Center for Advanced Reproductive Care considered the possibility of adding gestational carrier (popularly known as "surrogacy") services to our program. The IVF Ethics Committee considered practical realities and inherent ethical issues including the number of invested parties, significant economic costs, risks taken on by the gestational carrier, and the potential for free market exploitation. The Committee resolved that efforts to combat these concerns should include a push for industry self-regulation with restrictions on reimbursement, better professional guidance on this topic from professional organizations, collaborative research on program development,
\end{abstract}

trends, and outcomes, and uniform laws to protect all parties involved. With this in mind, the Committee and program physicians approved moving forward with careful institution of this program in collaboration with handpicked surrogacy agencies.

1. Department of Obstetrics and Gynecology, Division of Reproductive Endocrinology and Infertility, University of lowa Health Care, lowa City, IA 52242

2007 marked the $20^{\text {th }}$ anniversary of the first in vitro fertilization (IVF) cycle performed at the University of Iowa's Center for Advanced Reproductive Care (CARC). The program has grown remarkably during that time, expanding from 30 cycles of IVF in 1987 to 473 cycles resulting in 199 live births in $2007 .^{1} 2009$ also marked our $5,000^{\text {th }}$ oocyte (egg) retrieval. This growth is consistent with the increase in United States IVF treatments as a whole. Between 1996 and 2006, the number of IVF cycles has more than doubled in this country, growing from 64,681 per

Please cite this paper as: Ryan G. Establishment of a Clinical Gestational Carrier Program: Medical, Ethical, Legal and Policy Issues. Proc Obstet Gynecol, 2010 Apr;1(1):Article 8 [16p.] Available from: http://ir.uiowa.edu/pog/vol1/iss1/8/. Free full text article.

Corresponding Author: Ginny Ryan, Department of Obstetrics and Gynecology, University of lowa Health Care, 200 Hawkins Drive, lowa City, IA 52242. Phone, 319-384-9170. Email: ginnyryan@uiowa.edu, 
year to 138,198 per year. ${ }^{2}$ The number of IVF live birth deliveries has increased more than two and a half times during this same period, from 14,507 per year to 41,343 per year. $^{2}$ It is estimated that $1 \%$ of children now born in the United States were conceived through IVF. ${ }^{2}$

This expansion is due to a number of factors, including increased availability and exposure of IVF, scientific and technological improvements that make IVF a viable option for more patients, increased acceptance of non-traditional family building, and a trend in delaying childbearing (which increases the number of infertile women). Besides a general increase in demand for IVF treatment at the University of lowa over the years, the program has expanded to include an embryo donation program in 1997 (for embryos already created through IVF and subsequently cryopreserved), treatment of lesbian couples in 2004, and treatment of single women in 2007.

With pressures to expand, advancing technologies, and increased public exposure comes a need for ongoing evaluation of the medical, ethical, and legal proprietary of IVF treatments offered. This is particularly true when the traditional definition of "family" is challenged by the composition of our patient populations.

For this reason, each of the above mentioned changes at the CARC was preceded by consultation with the hospital's legal advisory team, an invitation for comments and concerns from local care providers, and consideration by an IVF ethics committee.
In 1999, members of the IVF ethics committee published an account of the establishment of CARC's embryo donation program. ${ }^{3}$ This paper describes how faculty members responded to the problem of expanding numbers of cryopreserved embryos in storage by investigating worldwide practices, surveying patient desires regarding the disposition of their cryopreserved embryos, and charging an ethics committee with addressing the ethical and policy questions involved in establishing such a program. The publication provides the IVF community with an example of how to ethically tackle new problems arising in this unregulated field.

This practice of careful consideration prior to program expansion proved invaluable this past year as we contemplated adding gestational carrier services (popularly described as "surrogacy" services) to our CARC treatment options. This service represented the only nonexperimental IVF treatment we did not offer; in contrast, $81 \%$ of 426 IVF centers reporting 2006 data to the CDC do offer gestational carrier treatments. ${ }^{2}$

Without these services, women or couples with a significant uterine infertility factor and gay men are excluded from infertility treatment and the option of having genetically related offspring.

Despite the availability of this treatment option in the United States, there is a relative dearth of practical guidance available in the literature or through the country's professional organizations on how to appropriately establish a gestational carrier program. 
The American Society for Reproductive Medicine (ASRM), the country's primary professional organization for reproductive specialists, has nothing in their Ethics Committee Reports, Technical or Educational Bulletins, Position Statements, or Guidelines specifically addressing this topic. The issue is only mentioned briefly in a report entitled "Family members as gamete donors and surrogates." ${ }^{4}$ In this report, the ASRM comments that use of family members as surrogates may be ethically acceptable if careful counseling is provided and intergenerational surrogacy is closely scrutinized.

The American College of Obstetricians and Gynecologists (ACOG) issued its first Committee Opinion on "surrogate motherhood" in 1983 and most recently updated the statement in February, 2008. ${ }^{5}$ They acknowledge that this practice "continues to be controversial" and that "considerable disagreement persists within the medical profession, the medical ethics community, state legislatures, the courts, and the general public." The statement allows that "a physician may justifiably decline to participate in initiating surrogacy arrangements for personal, ethical, or medical reasons," but does list expected responsibilities for those obstetricians and gynecologists who choose to assist with these treatments.

ACOG suggests that surrogacy arrangements are best "overseen" by private nonprofit organizations, though they acknowledge that most surrogacy organizations are entrepreneurial in nature and thus must be closely investigated by the responsible physician. They further counsel infertility specialists that the intended parents and surrogate mother should be appropriately "screened" (apparently medically) and provided with independent mental health counseling and legal representation. Finally, infertility specialists are "encouraged to participate in research that is intended to provide data on the outcomes of surrogacy arrangements." ${ }^{5}$

With these limited guiding principles, we set about to answer the challenge set by one of our IVF ethics committee members who wrote, "Just because we can do this doesn't mean we should." In other words, we first wanted to determine whether we had the medical, legal, economic, and psychosocial means to establish a successful gestational carrier program in lowa. Secondly, though not necessarily separately (since a poorly administered program cannot be ethically viable), we wanted to determine whether the balance of medical need, community support, legal acceptance, libertarian ideals of procreative liberty, and risks of harm tipped in favor of establishing this program. A description of this factfinding mission and decision-making process follows.

\section{The Basics of Gestational Carrier (Surrogacy) Arrangements}

While this phenomenon of carrying a child that you do not intend to raise appears at first to be a modern one, there are actually two surrogacy arrangements documented in the book of Genesis in the Old Testament of the Christian Bible. In chapter 16, Abraham and his infertile wife, Sarah, ask her maid, Hagar, to bear Abraham's children. And in chapter 30, Isaac and 
his infertile wife, Rachel, ask the same of her maid, Bilbah.

What modern science has wrought, however, is an increasingly complex and intentionally non-traditional definition of family. As Liza Mundy comments in her investigative report Everything Conceivable, "science has given us something new: families that are designed, from the start, to have only a single parent; to have quite a few parents; to have two parents, only one of whom is biologically related to the child, the other of whom is biologically unrelated, with a third party out there who is biologically related but, often, unknown. Families with these qualities have spontaneously arisen in the past, and still do, of course [by war, adultery, epidemics, childbed fever, remarriage, slavery, stepparents, social upheaval, and shifts in gender roles], but now they are being consciously formed."

as "Gestational carriers," also known mothers" pregnant, carry, and deliver a child under an agreement that the child will be raised by another woman or couple (known as the "intended," "social," "rearing," or "commissioning" parent(s)). ${ }^{6-8} \quad$ These surrogates may have no genetic relationship to the child they carry (sometimes referred to as "full surrogates" or "gestational carriers") or they can provide oocytes and therefore half of the child's genetic material (sometimes referred to as "partial" or "traditional" surrogates). For the remainder of this article, I will use the term "gestational carrier" (GC) when there is no genetic relationship between the fetus and gestating woman, "surrogate" when there is a genetic relationship, and "intended parent(s)" (IPs) for the person(s) who plan to raise the child.

Any reproduction involving three or more parties is termed "collaborative reproduction." To complicate this "collaboration" further, there may be more than three parties involved in "parenting" a desired child. The IP(s) may provide all or none of the gametes that make up the embryo. In an extreme case, the IP(s) may choose a male donor to provide sperm, a female donor to provide eggs, and a GC to carry and deliver the child. This type of reproductive "collaboration" thus includes five potential "parents."

There are important practical, ethical, legal, and policy implications of the differences between a surrogacy arrangement and a GC arrangement. Practically, surrogacy arrangements require only the placement of sperm in the vagina, cervix, or uterus of the surrogate, either by intercourse or artificial insemination. Gestational carrier arrangements require the medical ovarian stimulation of the female IP (or oocyte donor) with weeks of injectable medications and monitoring, surgical retrieval of oocytes, in vitro fertilization, and transfer of embryos into the uterus of the gestational carrier. The latter is more medically invasive and risky, requiring special medical expertise and facilities, and much more expensive.

Artificial insemination of traditional surrogates can be done at home, avoiding clinic costs and FDA (Food and Drug Administration)mandated lab testing costs. If done in a clinic setting, costs of insemination and 
lab testing may be less than $\sim \$ 1,500$. The IVF treatment that is required in GC arrangements, on the other hand, averages $\$ 9,000$ - $\$ 20,000$ per treatment cycle, depending on whether an oocyte donor is used and whether preimplantation genetic testing is performed. This may effectively "price out" many infertile person(s) from GC cycles.

\section{The Ethics and Practicalities of Surrogacy Law}

While surrogacy arrangements are more simple and affordable than GC arrangements, there are serious legal concerns that make using a surrogate more fraught than using a GC. Because a surrogate is the genetic mother of the child in question, if she changes her mind it is very likely that the court can deem a surrogacy contract unenforceable and award custody of the child to the surrogate. The courts tend to appeal to the standard of the best interest of the child in these cases.

As lawyer and bioethicist John Robertson points out, "the question of enforcing preconception surrogacy agreements for rearing is the most controversial issue in collaborative reproduction." ${ }^{9}$ Highly publicized cases of surrogates refusing to fulfill their agreed upon obligations have not helped to sway the courts towards enforcement. ${ }^{9}$ This was true in the Baby $M$ case in New Jersey in 1988 (In The Matter of Baby M 109 NJ 396 (1988)) where the New Jersey Supreme Court deemed the pre-birth agreement unenforceable, named the surrogate as the child's "natural mother," and awarded custody of the child to the surrogate despite the fact that the child had lived with her IPs for two years.

Because of such risks, most collaborative reproductive arrangements now preclude such traditional surrogacy, and most clinics and agencies do not provide these services. Early on in the process of considering adding surrogacy treatments to our program - prior to any discussion by the IVF ethics committee a decision was made to avoid such legal custody and contract battles, along with the potential for the greater difficulty a surrogate might have in relinquishing parentage, by restricting ourselves to considering GC treatment cycles only. Nevertheless, even these arrangements are legally muddy, with no unified body of law to which to appeal.

As attorney Mark Johnson writes in his publication Checklist on the Law of Surrogacy, "traditional "laws on the books' concerning paternity, maternity (if these laws address that issue at all),... issuance of birth certificates, parental rights and obligations, informed consent for medical procedures, 'Roe v. Wade' questions, medical insurance, infectious disease, pre- and post-natal support obligations, to name but a few, are wholly inadequate." ${ }^{10}$ Gestational carrier arrangements have not been the topic of any generally adopted "uniform acts," although the organization known as the "Uniform Law Commissioners" has drafted just such a "Uniform Status of Children of Assisted Conception Act" $^{\prime 11}$ in an attempt to remedy this situation. In the absence of general acceptance of any such a uniform state law, however, laws have been determined on a state by state basis and vary markedly. 
New York, for example, deems surrogacy contracts void and unenforceable, ${ }^{12}$ while Texas courts will specifically approve and enforce gestational carrier arrangements to protect all parties involved. ${ }^{13}$ lowa, on the other hand, is like most states: there are no laws providing guidelines or procedures for surrogacy arrangements. ${ }^{14}$ In such states, it is unclear what might happen if a GC breaches her contract or whether a new law severely restricting these practices might be enacted at any given time. lowa Code 70.11, which states that it is unlawful to sell or purchase another person, does contain a specific exception for surrogacy arrangements. Otherwise, adoption procedures are generally utilized to transfer parental rights to IPs in lowa and legislatively similar states.

lowa has drafted a Senate Study Bill (\#253) entitled, "The Human Reproductive Technologies and Surrogacy Act"15 to remedy this situation. In this Bill, maternity and paternity are granted to the surrogate I GC and her husband (if she is married) unless they "relinquish all rights and duties as parents of a child to be conceived through assisted conception." A written agreement to this effect must be approved by the court prior to conception, and the intended parents must be a married, heterosexual couple. This Study Bill was first introduced in 1996, but has yet to make it into law. Nationally, the American Bar Association produced a Model Act of Assisted Reproductive Technologies (ART) that provides an analysis of issues such as informed consent, counseling and consultation, privacy, the status of children of ART, payments to donors, heath insurance and professional liability. ${ }^{16}$ Again, however, this has not had a great deal of influence over state legislatures to this point.

Nevertheless, there are many lawyers in the United States that practice "reproductive law" and have a good understanding of the relevant statutes paramount to the success of gestational carrier arrangements. These lawyers are absolutely essential to successful negotiation and drafting of contracts for the parties involved, and for adjudication of maternity and paternity. As ACOG counsels, it is imperative that the $\mathrm{GC}$ and $\mathrm{IP}(\mathrm{s})$ are each assisted independently by separate knowledgeable representatives.

Points addressed in a contract between a GC (and her family) and IP(s) are best explored in conjunction with psychological as well as legal counsel. Even if not mandated by state law, contracts are essential and should include all expectations of all parties during the evaluation (including background checks and psychological testing, for example), medical stimulation (including amount of time the GC needs to be available), pregnancy (including what prenatal testing might be performed, what will happen if complications develop, and whether the GC would undergo an abortion for fetal abnormalities), delivery (including whether C-section is mandated for certain circumstances), and post-partum (including how communication will continue between parties and how birth certificates will be prepared). 
In our exploration of the legal aspects of GC arrangements in lowa, we discovered that practicing reproductive law specialists were not readily available in our local community. Furthermore, many of these subspecialists are found practicing in conjunction with, or as owners of, surrogacy agencies. It became clear that the most appropriate way to find well-trained legal representation for our patients was to turn to these agencies for assistance. Since there are no gestational carrier / surrogacy agencies in the state of lowa, so we turned our attention to neighboring Midwestern states.

\section{The Realities and Ethics of the Business World of Surrogacy}

Most IVF programs that offer
oocyte (egg) donor treatments or gestational carrier treatments use private, for-profit agencies to advertise for donors / carriers, screen and match potential candidates, and broker financial and other arrangements between the parties. While the University of lowa CARC has been offering oocyte donor treatment for many years, we have been in the minority of programs that provide all of these services in-house. This has allowed us to maintain control over all aspects of the program, guaranty high quality, and keep costs low for our patients.

Because of our lack of experience with egg donor agencies, however, we were unprepared for the infertility business world that became apparent in investigating surrogacy agencies. This for-profit world has resulted from the U.S. free market growing to meet the needs of the infertile population. It is difficult to know exactly how many egg donor or gestational carrier agencies exist at any given time in the United States, since there is no certification process or regulatory agency governing these businesses. ASRM does have a list of 87 egg donor agencies that have "signed an agreement with the Society for Assisted Reproductive Technology that states that they will abide by the ASRM Ethics Committee Guidelines governing the payment of egg donors."

Fifty of the 87 agencies also provide facilitation of GC cycles. The vast majority of these agencies are forprofit, and most of these appear to be owned by entrepreneurial women, with or without training in healthcare, who have experienced infertility or acted as a GC personally in the past. A smaller number of agencies have been started by, or in conjunction with, reproductive lawyers with experience negotiating gestational carrier contracts and parentage. Several describe their agencies as "boutique," and a few have specifically developed to assist subpopulations such as Asian immigrants, Orthodox Jews, or international IP(s).

It is not a surprise that costs to IPS and reimbursement of GCs vary widely, given the entrepreneurial nature of the business, the lack of regulation, the variety of locations and clients, and the uncertainty surrounding the appropriate level of compensation for gestational carriers. GCs who do not ask for compensation (beyond payment for medical bills and insurance, shortterm life insurance, legal fees, and expenses) are known as "compassionate" or "altruistic" carriers, 
and are often family members or friends of the IPs. "Compensated" GCs, by far the most common in the United States, also receive payments that are distributed throughout the pregnancy. The total amount of this "compensation" seems to vary from $\sim \$ 18,000-\$ 30,000$, and is higher if the carrier has successfully provided the service in the past. Reimbursement may also be higher if the carrier is pregnant with multiples, if she has a C-section, if she requires an invasive prenatal procedure, or if she has lost wages due to her fulfillment of the gestational carrier role.

A broad estimate of the costs of parenting with a gestational carrier and agency totals between $\$ 50,000$ and $\$ 150,000$, including payments to the GC, an egg donor (if necessary), brokering agencies, an IVF clinic, and lawyers, plus medical and life insurance for the GC, escrow fees, and travel expenses. Amazingly, approximately $1.4 \%$ of the 138,198 IVF cycles performed in 2006 included a GC, despite these exorbitant costs.

Reimbursement / compensation of GCs is one of the thorniest legal and ethical issues both in the United States and worldwide. It is this issue more than any other that has prompted both popular and legal rejection of contracted relationships between IPs and GCs, and lawyers must be careful to explore whether statutes against human "trafficking" may be applied when money changes hands in GC arrangements in a given state.

In Canada, the Assisted Reproduction Act of 2004 makes it a "criminal offense to pay or offer to pay a woman to act as a surrogate; to pay or offer to pay a person to arrange for the services of a surrogate; to advertise payment for surrogacy or the arrangement of surrogacy." ${ }^{17}$ Even reimbursing a surrogate for her expenditures during gestation is now a controlled act in Canada. Australia and the United Kingdom similarly permit altruistic, but not commercial, surrogacy. ${ }^{17}$

Certainly, monetary compensation raises ethical issues regarding protection of the GC's interests, body, and privacy, as well as protection of the intrinsic value of human life. Opponents of GC / surrogate use have described this practice as anywhere from "unsavory" to "immoral," since they believe that the gestating women are treated as means to an end rather than ends in themselves. ${ }^{7}$ There are concerns that a woman will be considered no more than a "womb for rent" and that her privacy and right to autonomous decision-making will be discounted. Furthermore, monetary conpensation may induce a woman to discount her physical or psychological risks or to conceal important medical information. ${ }^{7,18,19}$ Compensation also creates the potential for exploitation. In general, oocyte donors are selected based on beauty, intelligence, health, and youth, and gestational carriers are selected based on past reproductive performance, compliance, and willingness to dedicate a great deal of time and effort to their role. ${ }^{6}$ The latter woman is very often of lower socioeconomic status than an egg donor or the IPs and is susceptible to economic exploitation. ${ }^{6}$

Increasingly common is a type of "reproductive tourism" in which relatively 
wealthy infertile couples send their embryos to be gestated by foreign women for a fraction of the cost of this practice in the United States. ${ }^{20,21}$ India is currently the favored location for this practice, where surrogacy is legal and clinics have arisen specifically to cater to these "tourists." Reports from India are surfacing that suggest gestational carriers are inadequately informed of the process and risks, and that many are illiterate..$^{20,21}$ All of this adds to the aura of injustice surrounding the business of gestational carrier arrangements.

Remuneration related to transferring embryos and newborn children may also imply that they are commodities to be bought and sold, and this is counter the value of, and intrinsic respect for, human life. ${ }^{18}$ Some compare this situation to adoption (where it is unacceptable to pay women to "adopt out" their children) or to organ donation (where there is a prohibition against paying someone to donate organs or tissue), and wonder how it can be ethically acceptable to pay gestational carriers.

On the other hand, it is ethically acceptable to pay research subjects for their time, inconvenience, and discomfort. There is no question that IVF treatments and a 40-week pregnancy, with its attendant physical, emotional, and psychological risks and challenges, represents an sacrifice of time, convenience, and comfort for the GC. It is also fair to compare the challenges of the gestational carrier's experience to other positions of employment, where time, effort, risktaking, care, and talent are remunerated. The common practices of distributing payments to GCs over the course of the pregnancy, instead of in one lump sum after the child is delivered, and of compensating experienced GCs more than first-time GCs, are consistent with this conceptualization.

Nevertheless, these ethical concerns related to compensation become more and more appropriate as GC payment amounts increase. For this reason, the unregulated nature of the free market system in the United States should be tempered by practical guidelines or oversight from biomedical ethics experts and professional organizations. While these do not exist currently, similar guidelines were developed by the ASRM Ethics Committee for financial compensation of oocyte donors. In this report, the Committee concludes that, "compensation should be structured to acknowledge the time, inconvenience, and discomfort" of the medical treatments, and that, "compensation should not vary according to the planned use of the oocytes, the number or quality of oocytes retrieved, the number or outcome of prior donation cycles, or the donor's ethnic or other personal characteristics." ${ }^{\text {18 }}$

The fact that for-profit agencies facilitate many of these treatments in the United States is also counter to the research mandate that good medical practice demands, and many commentators feel that IVF treatments using surrogates and GCs has proceeded without adequate preliminary experimental scientific study. ${ }^{5,22}$ Agencies that are competing for GCs and IPs are not likely to feel much scientific responsibility or to ask their clients to take part in studies, however. 
As Reproductive Endocrinologist Dr. Howard Jones writes, "...let's face it entrepreneurial business has a different ethic and goal than science." ${ }^{22}$

It is nevertheless true that agencies are in a unique position to provide the best quality care for infertility patients who need GCs to build a family. The very fact of their existence suggests that clinical IVF programs have not generally have the staff, experience, or connections necessary to efficiently screen and match GCs, coordinate logistics, arrange medical treatments, facilitate legal representation, and support very complex and fragile human relationships during a pregnancy. Agencies established by prior intended parents or gestational carriers have blossomed because these individuals saw a void that could be filled.

Our IVF Ethics Committee carefully considered developing a gestational carrier program completely in-house vs. working with established agencies. In the end, the decision was made to provide the medical assessments and treatments ourselves while working with a short list of wellrespected agencies that already have the staff and experience to facilitate coordination of recruiting, matching, payment, and legal contracts. One of the most compelling reasons behind this decision was concern about the daunting task of supporting all the involved parties throughout a long treatment and pregnancy process. This would require time, skills and personnel that we did not have in place. Secondly, we were uncertain of the level of need for these services in our community and it seemed imprudent to develop a complicated new program of services if they would be used only rarely. Finally, prevailing legal opinion suggested that it would be wise to transfer the legal responsibilities of contract negotiation and parentage agreements away from the purview of the hospital in order to protect the institution from litigation.

Nevertheless, this decision meant giving up the quality control and cost containment that we have with our oocyte donation program. For this reason, the ethics committee felt it vital to closely investigate the agencies with which we would collaborate. This was done through Internet research, phone conversations, and visits from agency representatives for presentations and face-to-face meetings. Most importantly, the Committee felt that our CARC's medical inclusion and exclusion criteria for IPs and GCs must be consistent with, or be allowed to trump, the criteria laid out by the collaborating agencies (see Box 1).

\section{The Clinical and Medical Ethics Issues in IVF Gestational Carrier Treatment}

Some on the ethics committee argued as a matter of gender equity and libertarian ethics that any woman should be able to decide against gestating and delivering her child and contract with a competent and informed gestational carrier, regardless of this intended mother's physical or medical ability to carry the child herself. The prevailing committee opinion, however, was in line with the ACOG Committee Opinion which states that, "because of the risks inherent in surrogacy arrangements, such arrangements should be considered only in the case of infertility 
or serious health-related needs, not for convenience alone. ${ }^{5}$

There are a number of medical reasons why someone may be unable to carry and/or deliver a child, or may be counseled against this. Congenital abnormalities or scarring may leave a woman's uterus unable to carry a pregnancy to term. Recurrent pregnancy losses without an apparent genetic link, especially those occurring in the second trimester, may also be an indication for using a gestational carrier. Pregnancy is impossible for women who have undergone hysterectomy at a young age for such issues as uncontrollable bleeding or endometrial or cervical cancer, and pregnancy is medically contraindicated in women with serious heart or lung abnormalities, with spinal cord injuries, or with significant bleeding or clotting disorders. A widower with cryopreserved embryos genetically related to his deceased partner may wish to gestate these embryos and raise the offspring. Finally, gay men require a gestational carrier or surrogate to build their genetically related families.

The CDC reports that 1,042 fresh non-oocyte donor IVF cycles in 2006 involved gestational carriers (approximately $1.4 \%$ of all such cycles). An unknown additional number of oocyte donor IVF cycles involved GCs, including those with gay male intended parents (a growing minority of IPS). Interestingly, the success rates of GC cycles in all age groups were better than those in which the intended mother carried the pregnancy. That is not to say, however, that relatively high pregnancy rates represent a medical reason to use GCs for more IVF cycles.
Though the CARC had been approached by several prospective IPs, and had assisted with clinical monitoring of local GCs being treated by other clinical programs, we were unaware of a significant demand in our local population for GC services when we began our investigation. Simply speaking to local obstetrics and gynecology providers about our plans to consider starting a GC program brought a flurry of interest from potential IPS, however. Current or past IVF patients who might benefit from GC treatment (such as those with recurrent failed IVF cycles or those who had remaining cryopreserved embryos from cycles prior to hysterectomy) learned of the program and got on a waiting list. These developments, in the absence of any focused advertising of the program, convinced us that there is a desire for these services in our community.

Because it is difficult to anticipate all possible medical indications for GC use, the ethics committee agreed that acceptance into the program must be done on a case by case basis with the consensus of all physicians in the program. Clinical inclusion and exclusion criteria developed by the IVF team and ethics committee are listed in Box 1. Developing this list prompted further ethics committee discussion regarding the social vs. medical value of setting age limits for parenting. While this question was not definitively answered, a consensus was reached that we would be consistent with our existing guidelines for donor oocyte and cryopreserved embryo recipients, for whom treatment ceases at their $50^{\text {th }}$ birthday. Possible exceptions could be brought to the clinical team on a case by case basis. 
In our discussion of how nontraditional families fit into the criterion of medical necessity, there was relatively limited debate over whether single men or gay male couples could be described as having a medical vs. social need for a gestational carrier. In the end, there was general agreement that these IPs would be accepted into the program. Perhaps the willingness to offer GC services to the single and gay male population can be attributed to the general acceptance of current treatments of single and lesbian women and couples or to a general sociocultural acceptance of male parenting. The committee also discussed the fact that there is no current evidence that marital status or sexual orientation affects one's ability to parent or one's interest in childbearing and rearing.

Lawyer and bioethicist John Robertson articulates the primacy of procreative liberty in Children of Choice: "procreative liberty [the freedom to decide whether or not to have offspring] is a deeply held moral and legal value that deserves a strong measure of respect in all reproductive activities," and this value "should be equally honored when reproduction requires technological assistance," ${ }^{\prime 9}$ Indeed, medical procedures such as IVF open up choices to many infertile people otherwise unable to fulfill their fundamental desire to have a genetic family, and gestational carrier arrangements allow us to avoid further discrimination against single men or gay male couples. Our ethics committee's decision was also in line with the ASRM Ethics Committee recommendation that "programs should treat all requests for assisted reproduction equally without regard to marital status or sexual orientation. ${ }^{23}$

\section{Risks of Harm to Gestational Carriers and Offspring}

As bioethicist Barbara Berg notes, "the acceptance of medical interventions to maximize the choices available to infertile women [and men] is not the same as uncritically accepting the medicalization of reproduction." Despite the fact that GCs are used ultimately for the same ends as standard IVF - to help an infertile person or couple produce offspring to rear - we must acknowledge that inserting a very invested third party into the process results in unique, and often very controversial, issues and risks.

Medically and ethically, the GC must be treated as an independent patient in this process. This important fact is at risk of being overlooked by the surrogacy agency, whose fees are coming from IP(s), and by the IVF clinics, which are first approached for treatment by the IP(s). It is vital that the GC has her own legal representative (as mentioned above) to ensure her interests are represented in the contract negotiations, that she is fully informed, consented, and treated objectively by the IVF clinic, that she has an independent obstetrician who acknowledges her autonomy to make medical decisions during the pregnancy, and that she has optimal psychological support throughout the process.

One of the most important elements that must be in place to optimally prepare a GC for her upcoming experience, and to support her throughout the process, is an 
experienced health psychologist. GCs must meet with the psychologist both independently from their IP(s) and then together with them. An experienced and talented psychologist can pick up on risky issues and can give the GC a way to opt out of the process. He or she can help make the optimal match between the involved parties based on characteristics and expectations.

While these efforts may represent the best possible way to maintain respect for a GC's rights, Barbara Berg echoes a common concern that "the notion that a woman can predict her level of attachment to her fetus and should be held to a previously signed contract is unacceptable." ${ }^{19}$ Indeed, pregnancy is a profoundly life-changing event that may also risk the health and life of the gestating woman (there were 569 maternal deaths in the United States in 2006). It is difficult to imagine how one can fully inform a potential GC of the complexity of her upcoming experience.

On the other hand, some have argued that "surrogacy is, at least potentially, an example of what feminist bioethicist Barbara Katz Rotheman calls 'reproductive communism,' in which women help each other through all stages of childrearing depending on their desires and abilities." ${ }^{6}$ While it may be difficult to fully inform a potential GC what she is about to undertake, assuming that she cannot make a rational decision about her reproductive intentions may be equally unacceptable. As John Robertson points out, "rejection of preconception intentions to fix postbirth rearing rights and duties seems to be based on paternalistic attitudes toward women or on a symbolic view of maternal gestation."

In an effort to maintain consistency and quality control of our GC program, the ethics committee initially proposed that all GCs and IPs be screened by our program's health psychologist. We came to discover, however, that our chosen agencies have health psychologists with markedly more experience in this arena and with connections to the Mental Health Professionals Group of the ASRM. Furthermore, it became clear that it is much more practical to use health psychologists located near the agency offices so that these specialists can facilitate meetings between the GCs and IPs and can offer support during the pregnancy by way of communication with the agency representatives.

Perhaps the most concerning issue in this debate is that of potential harms to the offspring or other existing children affected by these treatments. There are no good data regarding risks and benefits to the children resulting from GC or surrogacy arrangements $s^{5,8}$, and yet they are the only persons who have not elected to be involved. Certainly, years of legal battles over custody can be assumed to be harmful and thus effective psychological screening of all parties and good legal representation are vital to avoiding this situation. Others suggest that "proper experimental design and proper data collection, followed by rigorous analysis and peer review"22 of family outcomes should be undertaken before these services are expanded any further. It is interesting to weigh any potential harm to the offspring against the alternative of 
non-existence if the reproductive assistance had not been provided at all.

\section{Conclusion}

The issues surrounding gestational carrier arrangements in assisted reproductive care are truly unique: the number of invested parties, the significant economic costs, the extraordinary risks taken on by the GC, and the multiple contentious ethical dilemmas, such as concerns regarding the free market response to the growing infertile population. Adequately balancing the rights of all involved parties with an individual patient's procreative liberty right requires careful planning and application of policies. While it is possible to do this successfully, it is not surprising that some IVF programs have found they cannot adequately meet these challenges and thus cannot offer appropriate GC services to their patients. The number of these programs is decreasing, however.

When presented with this challenge in our IVF program, we approached our IVF ethics committee with some background information and asked them to consider the question of whether we could, and should, expand our treatment options to include GC treatments. Interestingly, we found that the question of practicalities (the "could") was more contentious and difficult to resolve than the question of ethical propriety (the "should"). Perhaps the reason for this lies in the current sociocultural and ethical climate in which privacy and libertarianism are valued, allowing wide latitude in the achievement of the goal of begetting children.
Into this wide latitude has jumped the business world of surrogacy agencies who take advantage of what we came to understand during our investigation - that the logistics of gestational carrier cycles are daunting and at the same time vital to administering an ethical program. While filling this important role, for-profit agencies have unfortunately added to the aura of commercialism, especially as fees and GC payments continue to rise. Efforts to combat these concerns should include a push for industry selfregulation, with restrictions on reimbursement.

We also came to conclude that IVF clinics would benefit from better professional guidance on this topic from the ASRM. Acknowledgement of this growing treatment area may help foster important collaborative research on program development, trends, and outcomes. And for courts and invested parties across the country, uniform laws dealing with this issue are far overdue. With these aforementioned details and future wishes in mind, our IVF ethics committee gave the approval to move forward with development and institution of a gestational carrier program in collaboration with handpicked Midwestern surrogacy agencies.

\section{References}

1. https://www.sartcorsonline.com/rptCSR PublicMultYear.aspx?ClinicPKID=1931

2. http://www.cdc.gov/ART/ART2006/508P DF/2006ART.pdf

3. Van Voorhis BJ, Grinstead DM, Sparks, AET, Gerard JL, Weir RF. (1999). Establishment of a successful donor embryo program: medical, ethical, and 
policy issues. Fertility and Sterility, 71(4):604-608.

4. The Ethics Committee, American Society for Reproductive Medicine. (2003). Family members as gamete donors and surrogates. Fertility and Sterility, 80(5):1124-1130.

5. ACOG Committee on Ethics. (2008). Surrogate Motherhood. Obstetrics and Gynecology, 111(2):465-470.

6. Mundy L. (2007). Everything Conceivable - How Assisted Reproduction is Changing Men, Women, and the World. New York, NY. Albert A. Knopf.

7. Steinbock B, Arras JD, London AJ eds. (2003). Ethical Issues in Modern Medicine $6^{\text {th }}$ ed. Boston, MA: McGraw Hill.

8. ESHRE Task Force on Ethics and Law. (2005). ESHRE Task Force on Ethics and Law 10: Surrogacy. Human Reproduction, 20(10):2705-2707.

9. Robertson JA. (1994). Children of Choice. Princeton, NJ: Princeton University Press.

10. Johnson MA. (1996). Checklist on the Law of Surrogacy. The Surrogacy Center, Inc.

11. www.nccusl.org/nccusl/uniformact sum maries/uniformacts-s-usocoac.asp

12. www.surrogacy.com/legals/article/nylaw. $\underline{\mathrm{html}}$

13. www.surrogacy.com/legals/article/txlaw. $\underline{\mathrm{html}}$

14. www.surrogacy.com/legals/article/ialaw. $\underline{\mathrm{html}}$

15. http://nxtsearch.legis.state.ia.us/NXT/gat eway.dll?f=templates\&fn=default.htm

16. Kindregan CP, Snyder SH. (2008). Clarifying the Law of ART: The New American Bar Association Model Act
Governing Assisted Reproductive Technology. Family Law Quarterly, 42: 203.

17. Reilly DR. (2007). Surrogate pregnancy: a guide for Canadian prenatal health care providers. Canadian Medical Association Journal 176(4): 483 - 485.

18. The Ethics Committee of the American Society for Reproductive Medicine. (2007). Financial compensation of oocyte donors. Fertility and Sterility, 88(2):305 - 309.

19. Berg, BJ. "Listening to the Voices of the Infertile." Class reading.

20. Subramanian S. (2007) Wombs for Rent. Maclean's, July 2.

21. http://www.asrm.org/Media/LegallySpea king/vol42no1.html

22. Jones, HW. (1991). Commentary on ACOG Committee Opinion Number 88, November 1990 - "Ethical Issues in Surrogate Motherhood".WHI, 1(3), 138139.

23. The Ethics Committee of the American Society for Reproductive Medicine. (2006). Access to fertility treatment by gays, lesbians, and unmarried persons. Fertility and Sterility 86(5):1333 - 1335. 
Box 1: Clinical inclusion / exclusion criteria for gestational carriers and intended parents participating in University of lowa's gestational carrier program

Intended parents:

1. must have a medical reason that the female cannot carry and/or deliver a pregnancy

2. single parents must be less than 50 years old, or a couple less than 100 years in total age (though exceptions may be made on a case by case basis)

3. must be willing to cryopreserve excess embryos that are not transferred in a fresh cycle

4. are allowed to limit the number of oocytes that are inseminated (though this should be discouraged)

Gestational carriers:

1. must be between 21 and 40 years old

2. must have had an uncomplicated pregnancy and delivery in the past, and parented that child

3. must have had no more than one C-section

4. must be a non-smoker with no active drug or alcohol abuse issues

5. must not provide the oocytes for the intended pregnancy (i.e. no traditional surrogacy)

6. must not have a history of an intimate relationship with either intended parent

7. must not be the daughter or niece of the intended parent(s) 